\title{
Enhanced M1 and Impaired M2 Macrophage Polarization and Reduced Mitochondrial Biogenesis via Inhibition of AMP Kinase in Chronic Kidney Disease
}

\author{
Cong Li Xiao Yan Ding Dong Mei Xiang Jie Xu Xiang Lan Huang Fan Fan Hou \\ Qiu Gen Zhou
}

Division of Nephrology, Nanfang Hospital, Southern Medical University; State Key Laboratory of Organ Failure Research, National Clinical Research Center of Kidney Disease, Guangzhou, China

\section{Key Words}

Macrophage • Polarization • Mitochondrial biogenesis • Chronic inflammation • Chronic kidney disease

\begin{abstract}
Background: Macrophage polarization plays a pivotal role in the process of inflammation which is common in chronic kidney disease (CKD). Macrophages polarization under the condition of CKD remains poorly understood. Here we tested the hypothesis that CKD promotes macrophage M1 polarization. Methods: A rat model of CKD was established by reduced renal mass (RRM). Polarization of macrophages was induced in ex vivo macrophages from RRM rats and cultured ones under the condition of uremic serum. The markers were evaluated by RT-PCR, western blot, and flow cytometer. Results: Our data showed that macrophages from RRM rats displayed enhanced $M 1$ and impaired $M 2$ polarization as revealed by increased M1 markers (tumor necrosis factor $\alpha$, IL-6, IL-12p40, nitric oxide) and decreased M2 markers (IL10, CD206, arginase activity) in response to LPS and IL-4 induction, respectively. Treatment with uremic sera in peritoneal and bone marrow derived macrophages from normal rats led to similar results. Moreover, macrophages from RRM rats and cultured under the condition of uremic sera had reduced mitochondrial biogenesis. The disturbed macrophage polarization and mitochondrial biogenesis were accompanied by reduced activity of adenosine monophosphate-activated protein (AMP)-activated kinase (AMPK). Enhancing activation of AMPK restored mitochondrial biogenesis and M2 macrophage polarization. Conclusion: These observations suggest that CKD disturbs macrophage polarization and mitochondrial biogenesis through inhibition of AMPK. This might provide a novel therapeutic strategy for intervention of chronic inflammation in CKD.

C. Li and X.Y. Ding contribute equally to this work.

Dr. Qiu Gen Zhou,

Division of Nephrology, Nanfang Hospital, 1838 North Guangzhou Avenue, Guangzhou 510515, (P. R.China)

E-Mail nfyyskzqg@163.com
\end{abstract}

KARGER 125 


\section{Cellular Physiology Cell Physiol Biochem 2015;36:358-372 \\ \begin{tabular}{ll|l} 
and Biochemistry & $\begin{array}{l}\text { DOI: 10.1159/000430106 } \\
\text { Published online: May 06, } 2015\end{array}$ & $\begin{array}{l}\text { C } 2015 \text { S. Karger AG, Basel } \\
\text { www.karger.com/cpb }\end{array}$ \\
\cline { 2 - 3 }
\end{tabular}}

\section{Introduction}

Chronic systemic inflammation is prevalent in patients with chronic kidney disease (CKD) and linked to the development of cardiovascular disease, as well as progression of CKD $[1,2]$. Its underlying mechanisms remains poorly understood.

Macrophages, which could undergo their phenotypical switch in response to the microenvironment signals [3-6], form an important component of the innate and adaptive immune response. Two major macrophage subpopulations, classically activated (M1 polarization) and alternatively activated (M2 polarization) macrophages, have been increasingly recognized. M1 polarization macrophages produce pro-inflammatory mediators, whereas M2 polarization produces high levels of anti-inflammatory molecules. Therefore, a failure to appropriately control macrophage polarization may have a role in the development of disease. For example, excessive M1 polarization in adipose tissue has been linked to metabolic disease [7]. The distribution of M1 and M2 polarization in human atherosclerosis has been shown to be related with plaque instability [8]. In an animal model of kidney disease, macrophage polarization has been reported to be associated with progression of renal lesion $[9,10]$. Whether and how CKD affects macrophage polarization remains poorly appreciated.

A controlled regulation of mitochondrial mass through either the biogenesis or the degradation of this organelle represents a crucial step for proper mitochondrial and cell function [11]. Alterations of both mitochondrial mass and function are associated with several human pathologies, such as heart failure and metabolic diseases [11-13]. The metabolism of activated macrophages can undergo a dramatic change. A shift towards aerobic glycolysis occurs in M1 macrophages. Meanwhile, M2 macrophages exhibit oxidative metabolism with more limited rates of glycolysis $[14,15]$. In agreement, enhanced mitochondrial biogenesis promotes the M2 phenotype and attenuates macrophage-mediated inflammation. Conversely, inhibition of mitochondrial biogenesis limits the function of M2 macrophages, and greatly boosts the activating effects of lipopolysaccharide $[15,16]$. This information suggests that mitochondrial biogenesis plays an important role in the modulation of macrophage polarization.

Adenosine 5'-monophosphate-activated protein kinase (AMPK) senses cellular energy levels and regulates energy homeostasis. AMPK has been demonstrated to regulate mitochondrial biogenesis through activation of peroxisome proliferator activated receptor gamma coactivator-1(PGC-1) in skeletal muscle cells $[17,18]$. In macrophages, AMPK has been shown to modulate their polarization $[19,20]$. Meanwhile, the activity of AMPK under the condition of CKD remains unclear.

In the present study, we tested the hypothesis that CKD promoted M1 macrophage polarization. Our data showed that macrophages derived from the rats with reduced renal mass (RRM), a model of CKD, displayed enhanced M1 polarization and impaired M2 polarization. Moreover, these cells had reduced mitochondrial biogenesis and activity of AMPK. Enhancing AMPK activation through pharmacological agonist or genetic overexpression restored mitochondrial biogenesis and M2 macrophage polarization. Thus, our results might provide a novel therapeutic strategy for prevention and treatment of chronic inflammation in CKD.

\section{Materials and Methods}

\section{Animal preparation}

Male Sprague-Dawley rats initially weighing 180-200 g (Southern Medical University Animal Experiment Center) were used in this study and maintained under standardized conditions with a standard rodent diet. Rats were housed in temperature-controlled, light-cycled quarters with ad libitum access to food and water. The rats were subjected either to five-sixths nephrectomy by performing a right nephrectomy with surgical resection of two-thirds of the left kidney or to sham operation under anaesthesia. After 10- 


\section{Cellular Physiology Cell Physiol Biochem 2015;36:358-372 \begin{tabular}{l|l} 
DOI: 10.1159/000430106 & O 2015 S. Karger AG, Basel
\end{tabular} and Biochemistry Published online: May 06, 2015 www.karger.com/cpb \\ Li et al.: Macrophage Polarization in CKD}

14 wk of the surgery, rats were subjected to analysis. In the interventional study, rats were treated with intraperitoneal injection of 5-aminoimidazole-4-carbox-amide-1- $\beta$-d-ribofuranoside (AICAR, Calbiochem, San Diego, CA, USA) (500mg/kg body weigh daily for 1 week). The investigation conformed to the Guide for the Care and Use of Laboratory Animals published by the United States National Institutes of Health (NIH Publication no. 85-23, revised 1996). All animal procedures were approved by the Animal Experiment Committee of Southern Medical University.

Rats were fasted overnight $(12 \mathrm{~h})$, and blood was drawn by transcutaneous cardiac puncture after anesthesia. Blood was collected and centrifuged at $10,000 \times g$ to collect sera. Plasma creatinine levels were determined using commercial kits (Maker, Sichuan, China) according to the manufacturer's instruction.

\section{Cells culture and treatment}

Isolation and culture of peritoneal macrophages [16]. Peritoneal macrophages were obtained by a peritoneal washing with $10-20 \mathrm{~mL}$ cold Dulbecco's PBS. After centrifugation at $350 \times g$ for $5 \mathrm{~min}$, the cells were re-suspended in complete medium (RPMI 1640, 10\% FBS, 2 mM L-glutamine, 100 units/mL penicillin and 100 units/mL streptomycin) and incubated at $37{ }^{\circ} \mathrm{C}$ for $2 \mathrm{~h}$ in plastic culture plates. Then, the nonadherent cells were removed, and the adherent cells were cultured in complete medium.

Isolation and culture of bone marrow-derived macrophages (BMDMs) [16]. For BMDMs isolation, rats were sacrificed and both tibias and femur were collected. Bone marrow was flushed out the bones using a syringe filled with growth media. Bone marrow cells from the femurs and tibias were triturated in RPMI 1640 medium (Gibco Life Technologies, Carlsbad, CA, USA) containing $10 \%$ fetal bovine serum (FBS; Gibco). Red blood cells were lysed in lysis buffer $\left(0.15 \mathrm{M} \mathrm{NH}_{4} \mathrm{Cl}, 10 \mathrm{nM} \mathrm{KHCO}\right.$, and $0.1 \mathrm{mM}$ $\mathrm{Na}_{2}$ ethylenediaminetetraacetic acid, pH7.4), washed once in RPMI 1640 with $10 \%$ FBS, and then plated in RPMI 1640 supplemented $10 \%$ FBS and macrophage colony-stimulating factor (M-CSF). After 7-10 days in culture, nonadherent cells were removed and adherent cells were ready for experiment.

To determine the purity of rat macrophages, peritoneal macrophages and BMDMs were incubated with FITC-conjugated mouse anti- rat CD68 antibodies (AbD Serotec) prior to analysis by flow cytometer on fluorescence activated cell sorting (FACS) Caliber (Becton Dickinson, San Jose, CA, USA). Cells incubated with FITC-conjugated mouse IgG1 (an isotype control antibody) were used as blank control. The purity of isolated macrophages varied between $90 \%$ and $95 \%$ in six separate experiments (data not shown).

\section{M1 and M2 polarization [16, 21]}

For the polarization of M1 and M2 macrophages, lipopolysaccharide (LPS, $100 \mathrm{ng} / \mathrm{ml}$ ) (Sigma, St. Louis, MA, USA) or IL-4 (10 ng/ml) (PeproTech, Rocky Hill, NJ, USA) was added, respectively, in complete RPMI 1640 media for $24 \mathrm{~h}$. Then the function and the expression of M1 or M2 makers were assayed.

\section{Real-time RT-PCR}

Real-time RT-PCR was performed to evaluate the expression of M1- and M2-related factors. IL-6 and TNF- $\alpha$ were examined as M1-related factors while CD206 and IL-10 were examined as M2-related factors. Mitochondrial biogenesis related markers were also evaluated by real-time TR-PCR. Briefly, total RNA was extracted from cells and RNA was reverse-transcribed by using a reverse transcriptase. PCR was performed with SYBR green mix (Applied Biosystems, Foster City, CA) with the following PCR condition: $95^{\circ} \mathrm{C}$ for 3 minutes; $90^{\circ} \mathrm{C}$ for 20 seconds, and $60^{\circ} \mathrm{C}$ for 1 minute, for 40 cycles. Primers used in the study were shown in Table 1. Data were normalized to ß-actin.

\section{Cytokine Measurements}

Cytokines were measured from cell culture media using commercial enzyme-linked immuno sorbent assay (ELISA) kits from R\&D according to manufacturer's instructions. Cells were plated in 48- or 96-well plates. Levels of TNF- $\alpha$, IL-6, IL-12p40, and IL-10 in the supernatant were quantified.

\section{Western Blot}

Western blot was performed as previously described [22]. Following experimental treatments, cells were harvested, and lysed with lysis buffer. Protein concentration was measured with BCA protein assay reagent. The samples were diluted with lysis buffer, and equal amounts of protein were separated 


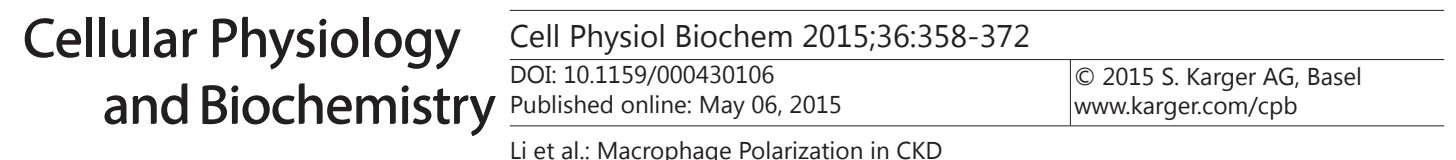

Table 1. Primers used in this study for PCR were shown

\begin{tabular}{lll}
\hline Gene & Forward & Reverse \\
\hline Rat TNF $\alpha$ & 5'-AACTCGAGTTACAAGCCCGTAG-3' & 5'-GTACCACCAGTTGGTTGTCTTTGA-3' \\
Rat IL-6 & 5'-GTGACCCT-GAGCAGTCCGTT-3' & 5'-GAAAGTGCAGCGCACCTGA-3' \\
Rat $\beta$-actin & 5'-GAAAGACGGCACACCCACC-3' & 5'-AAACCGCTTTTCCATCTTCTTCT-3' \\
Rat CD206 & 5'-GACAGATATGAACAAGCATTCC-3' & 5'- TGAACATCTGAGAGTCCTGTC-3' \\
Rat IL-10 & 5'-CAGACCCACATGCTCCGAGA-3' & 5'-CAAGGCTTGGCAACCCAAGTA-3' \\
Rat IL-12p40 & 5'-CTC CTG GTT TGC CAT CGT TT-3' & 5'-GGG AGT CCA GTC CAC CTC TA-3' \\
Rat iNOS & 5'-TCTCCACCAATGACCTGAT-3' & 5'-AAGTCAAATCCTACCAAAGTGA-3' \\
Rat Cyt B & 5-TGACCTTCCCGCCCCATCCA-3 & 5-AGCCGTAGTTTACGTCTCGGCA-3 \\
Rat NRF1 & 5-AAGCAAAGGGTTTCATGGAC-3 & 5-GGTACGAGATGGGCTATGCT-3 \\
Rat TFAM & 5-TCACACTGGTGACAGTTTCG-3 & 5-TAAAGCCCGGAAGGTTCTTA-3 \\
Rat Cyto C & 5-ACCAAATCTCCACGGTCTGTT -3 & 5-GGATTCTCCAAATACTCCATCAG -3' \\
Rat COX IV & 5-GTAACGCTACTCCGGGACAA-3 & 5-TCCAGGTAGTTCTGCCAACA-3 \\
\hline
\end{tabular}

by SDS-PAGE. The separated proteins were transferred to polyvinylidene difluoride membranes. The membranes were incubated with various primary antibodies. After washing, the membranes were hybridized with horseradish peroxidase-conjugated secondary antibodies (Santa Cruz Biotechnology). The blots were detected using ECL Plus Western Blotting Substrate. The relative signal intensity of bands was determined and standardized. The primary antibodies used in the study include anti-CD206 (1:500, SantaCruz biotechnology, CA, USA), anti-cytochrome C (1:1,000, Cell Signaling technology, Beverly, MA), anticytochrome C oxidase IV(1:1,000, Cell Signaling technology), anti-PGC1- $\alpha$ (1:500, abcam, Cambridge, UK), anti-PGC1ß (1:500, abcam), anti-acetylated lysine (1:1,000, Cell Signaling technology), p-AMPK (Thr172) $(1: 1,000$, Cell Signaling technology), AMPK $(1: 1,000$, Cell Signaling technology), and ß-Actin $(1: 1,000$, SantaCruz biotechnology).

Nitric Oxide (Griess) Assay [21]

Nitric oxide (NO) production was determined by measuring nitrite, a stable break-down product of nitric oxide metabolism, in cell supernatants. Supernatants were combined with an equal volume of $2 \%$ sulphanilamide (Sigma) and 0.1\% N-1-naphthylethylenediamine dihydrochloride (Sigma) and incubated at room temperature for $10 \mathrm{~min}$, and then the absorbance was measured at $550 \mathrm{~nm}$ in a microplate reader. Nitrite levels, as $\mu \mathrm{M}$ concentration, were determined based on a standard curve of known sodium nitrite concentrations.

\section{Arginase Assay}

Arginase activity in macrophages was assessed by measuring urea production, a by-product of the arginase reaction, as previously described [21]. Arginase activity is expressed as 1 arbitrary unit, where 1 unit equals the amount of enzyme needed to hydrolyse $1 \mu \mathrm{M}$ of arginine per minute. Arginase activity was determined based on a standard curve of known urea concentrations.

Mitochondrial mass Quantification

Mitochondrial mass was measured by fluorescence levels upon staining with MitoTracker Green FM (Molecular Probes) at $100 \mathrm{nM}$ for $25 \mathrm{~min}$ at $37^{\circ} \mathrm{C}$ [23]. Cells were then washed with PBS solution, detached, and resuspended in PBS solution containing1\% FBS for FACS analysis.

\section{Mitochondrial DNA Quantification}

Mitochrondrial DNA quatification was performed as previously [20]. To isolate mitochondrial DNA (mtDNA), cells were first homogenized and a mitochondrial fraction was isolated according to previously published protocols. Mitochondria were then lysed and mtDNA was then purified by phenol/ chloroform extraction and ethanol precipitation. Total DNA was isolated using standard protocols. We selected cytochromsome $B$ gene for quantification of mtDNA and $\beta$-actin gene for nuclear DNA (nDNA) quantification. The mtDNA copy number was evaluated by the mtDNA/nDNA ratio. Quantification of mtDNA and nDNA was performed by real-time PCR and analysis with a standard curve of a reference template. 


\section{Cellular Physiology Cell Physiol Biochem 2015;36:358-372

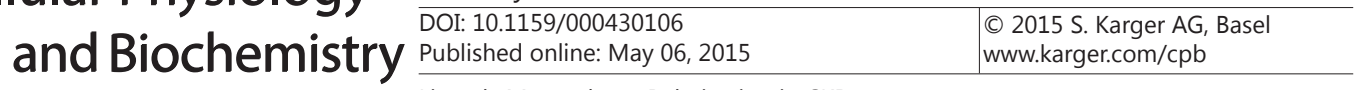 \\ Li et al.: Macrophage Polarization in CKD}

Immunoprecipitation for acetylated $P G C-1 \alpha$ and $P G C-1 \beta$

Acetylated-lysine PGC- $1 \alpha$ and PGC-1ß were analysed by immunoprecipitation of PGC- $1 \alpha$ and PGC-1ß from cells lysates with anti-PGC-1 $\alpha$ and anti-PGC-1ß followed by western blotting using an acetyl-lysine antibody.

Macrophage transfection

Macrophages transfection was performed as described previously [24]. Construction and preparation of the recombinant adenovirus was performed by Genechem Co. Ltd (Shanghai, China). The cDNA encoding residues 1 to 312 of $\alpha 1$ of AMPK, containing a mutation that alters threonine 172 to an aspartic acid, was used to construct the recombinant adenovirus. The cDNA of $\alpha 1^{312}$ was subcloned into the shuttle vector which was cotransformed with the supercoiled adenoviral vector into Escherichia coli. After selection, the recombinant adenoviral construct was transfected into the packaging cell line to be propagated. The adenoviral vector with no exogenous gene was used as a control. The recombinant adenovirus were purified and stored. Macrophages were transfected with empty vector or $\alpha 1^{312}$ adenovirus in serum-free medium for 2 hours. After the virus was removed, the cells were cultured for an additional 24 hours before further experiment. Transfection efficiency was $50.5 \% \pm 6.4 \%$, as determined by flow cytometric analysis of GFP expression in the cells cotransfected with CA 1 AMPK and pmaxGFP. Transfection was confirmed by analysis of expression of AMPK $\alpha 1$ with western blot.

\section{Statistical analysis}

Results were expressed as mean \pm SD of three or six independent experiments. All data were analyzed with SPSS 11.0 for Windows. Differences between two groups were assessed using unpaired two-tailed t-tests. Data involving more than two groups were assessed by analysis of variance (ANOVA). $P$ values $<0.05$ were considered significant.

\section{Results}

\section{CKD enhanced M1 and impaired M2 macrophage polarization}

The characteristic features of studied rats were shown in Table 2. Serum creatinine and blood urea nitrogen were significantly increased in RRM rats compared with sham rats, suggesting impaired renal function.

To evaluate the effect of CKD on macrophage polarization, we isolated peritoneal macrophage from rats with RRM. After 2 hours incubation, the adherent cells were stimulated with LPS and IL-4 for 24 hours, respectively, and the markers of M1 (TNF $\alpha$, IL-6, IL-12p40, and NO) and M2 (CD206, IL-10, and arginase activity) polarization were analyzed [25]. As shown in Figure 1 A, B, C, D, stimulation with LPS resulted in increased expression of TNF $\alpha$, IL-6, and IL-12p40 at both mRNA (Fig. 1 A1, B1, C1) and protein (Fig. 1 A2, B2, C2) levels, as well as increased expression of iNOS (Fig. 1 D1) and production of NO (Fig. 1D2), in macrophages from RRM rats compared with sham rats. The expression of TNF $\alpha$, IL-12p40, and IL-6, as well as production of NO, was also significantly increased under basal condition (incubation with medium alone) in macrophages from RRM rats compared with sham rats (Fig. 1 A, B, C, D), suggesting that RRM alone could promote M1 polarization. Conversely, expression of CD206 and IL-10 at mRNA (Fig. 1 E1, F12) and protein (Fig. 1 E2, E3, F2) levels, as well as arginase activity (Fig. 1G), was hardly response to IL-4 induction in macrophage from RRM rats compared with sham rats. The expression of these M2 macrophage markers was comparable under basal condition between sham rats and RRM rats, suggesting that RRM alone could not induce M2 polarization.

Table 2. Biochemical and physical parameters in the experimental rats $(\mathrm{n}=6) .{ }^{*} \mathrm{P}<0.05$ vs sham

\begin{tabular}{lcll}
\hline & $\begin{array}{c}\text { Body weigh } \\
(\mathrm{g})\end{array}$ & $\begin{array}{c}\text { Serum creatinine } \\
(\mu \mathrm{mol} / \mathrm{L})\end{array}$ & $\begin{array}{c}\text { Blood urea nitrogen } \\
(\mathrm{mmol} / \mathrm{L})\end{array}$ \\
\hline sham & $561.6 \pm 10.3$ & $46.4 \pm 5.2$ & $6.61 \pm 0.3$ \\
RRM & $439.1 \pm 11.5^{*}$ & $101.3 \pm 8.2^{*}$ & $17.9 \pm 0.4^{*}$ \\
\hline
\end{tabular}




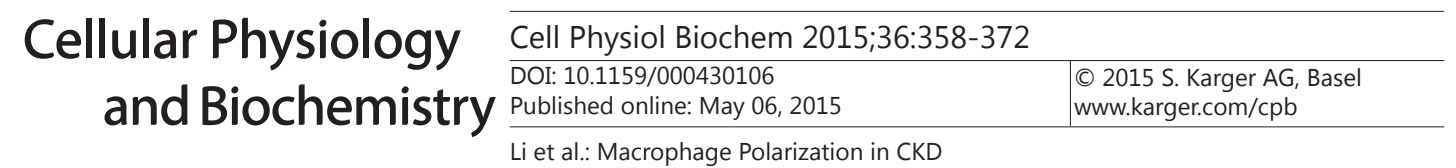

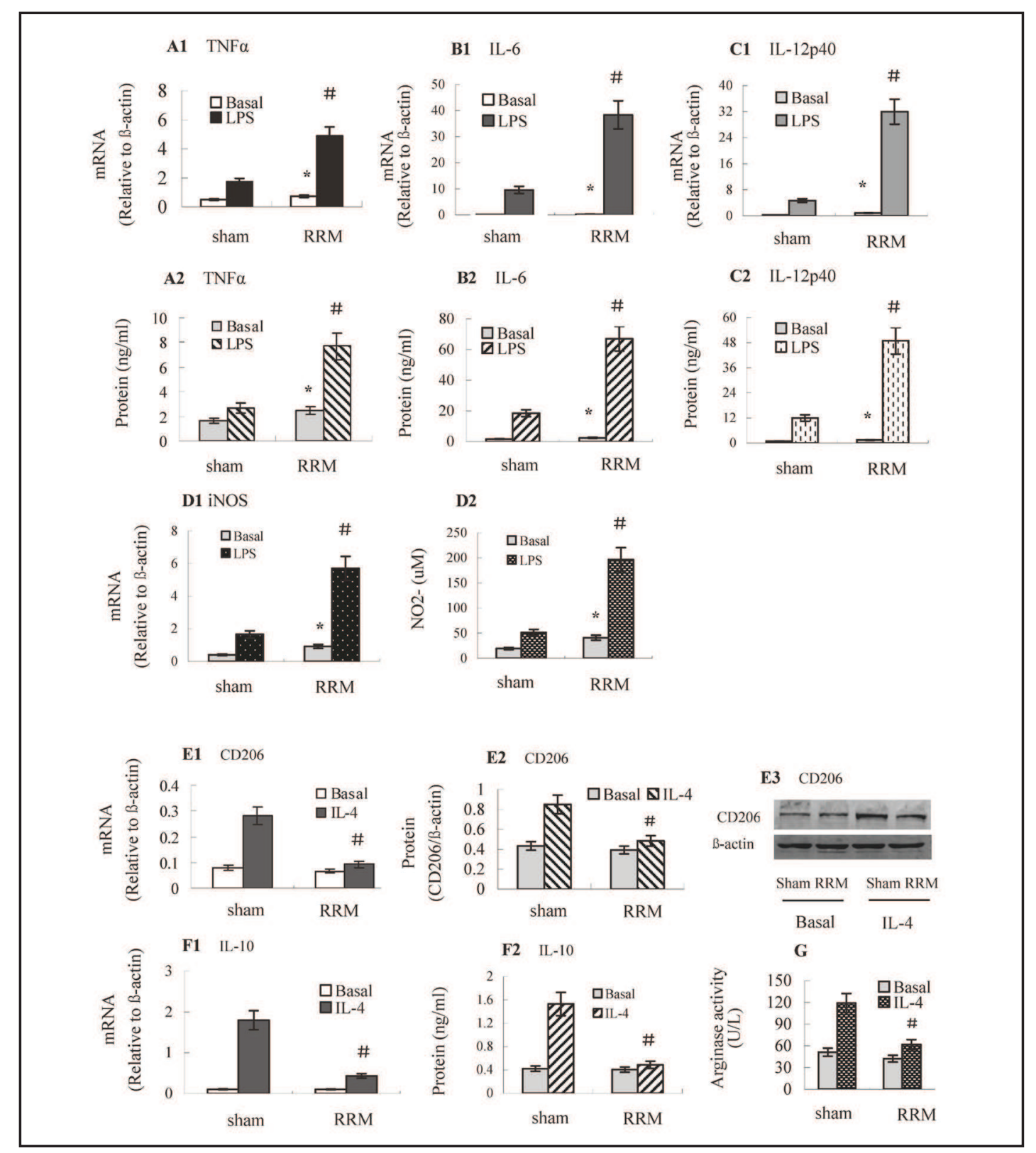

Fig. 1. Polarization of peritoneal macrophages from RRM rats. Peritoneal macrophages were isolated from RRM rats. After 2 hours incubation, the adherent cells were stimulated with LPS and IL-4 for 24 hours, respectively. The markers of M1 (A, B, C, D) and M2 (E, F, G) polarization were evaluated. Incubation with medium alone was referred as basal condition. Expression of TNF $\alpha$ (A1 and A2), IL-6 (B1, B2), and IL-12p40 $(\mathrm{C} 1, \mathrm{C} 2)$, as well as the expression of iNOS (D1) and production of NO (D2), were significantly increased in response to LPS stimulation in macrophages from RRM rats compared with sham rats. Meanwhile, expression of CD206 (E1, E2, E3) and IL10 (F1, F2), as well as arginase activity (G), were hardly response to IL-4 induction. E3: Representative products of western blot analysis for CD206. All bar graphs reported mean \pm SD for six experiments performed in triplicate except E1 and E3 (three experiments). ${ }^{*} p<0.05 v s$ sham under basal condition, ${ }^{*} p<0.01$ vs sham under LPS or IL-4 stimulation.

To further examine the effect of CKD on macrophage polarization, bone marrow derived macrophages were isolated from normal rats and incubated with sera derived from RRM rats to simulate the microenvironments of CKD. The cells were incubated with RRM rats sera (v/v 20\%) for 24 hours and then the markers of M1 and M2 polarization were analyzed KARGER 


\section{Cellular Physiology Cell Physiol Biochem 2015:36:358-372 \begin{tabular}{l|l} 
DOI: 10.1159/000430106 & $\begin{array}{l}\text { O 2015 S. Karger AG, Basel } \\
\text { www.karger.com/cpb }\end{array}$ \\
\cline { 2 - 2 } aublished online: May 06, 2015 &
\end{tabular}

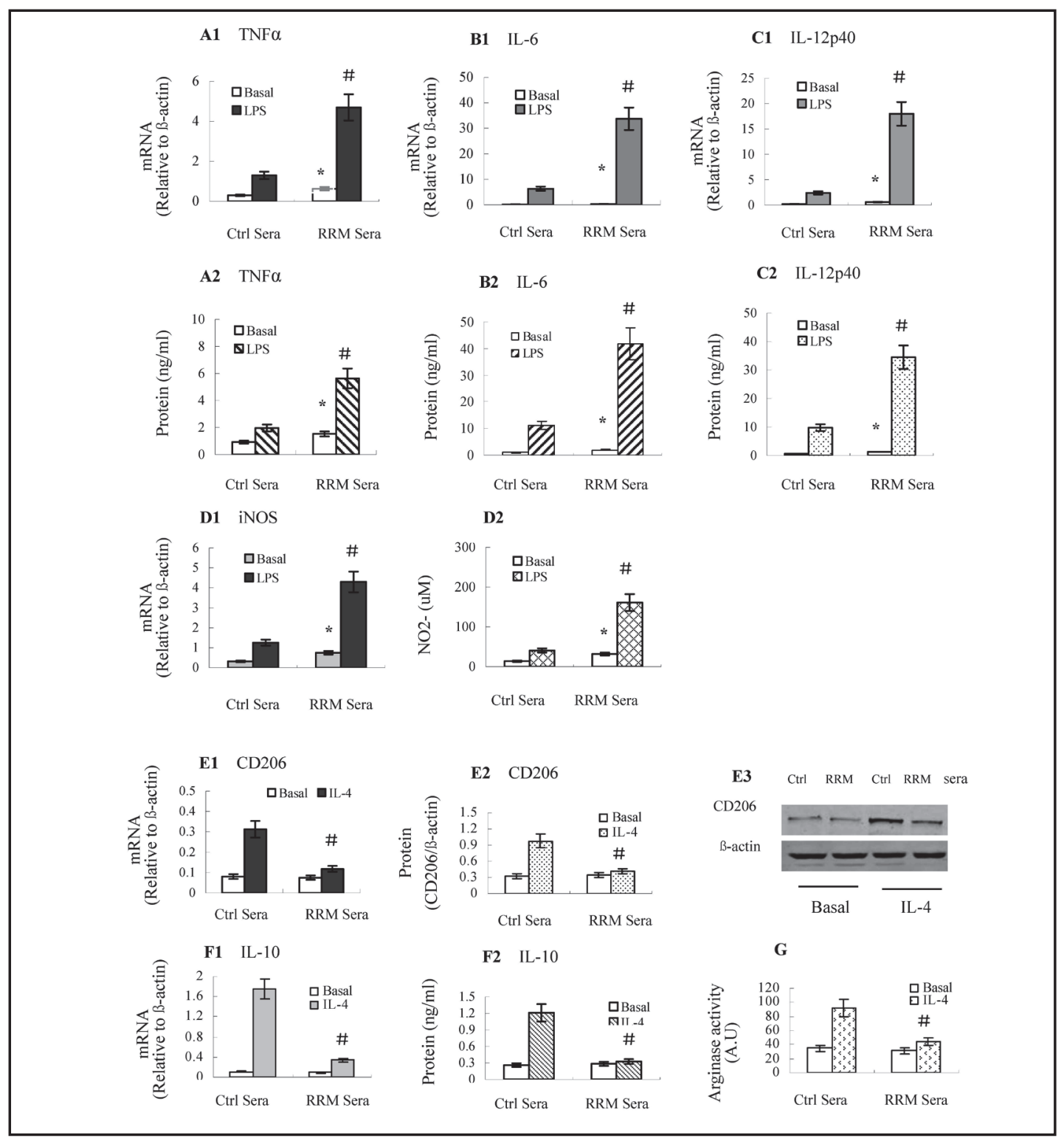

Fig. 2. Polarization of BMDM cultured with RRM rats' sera. Bone marrow-derived macrophages (BMDM) were isolated from normal rats. The cells were incubated with $20 \%(\mathrm{v} / \mathrm{v}$ ) sera from RRM rats for 24 hours. After washing with fresh medium, the cells were then stimulated with LPS and IL-4 for 24 hours, respectively. The markers of M1 (A, B, C, D) and M2 (E, F, G) polarization were determined. In BMDM treated with RRM rats' sera, expression of TNF $\alpha$ (A1, A2), IL-6 (B1, B2), and IL-12p40 (C1, C2), as well as the expression of iNOS (D1) production of NO (D2), was significantly increased in response to LPS stimulation compared with control sera. Meanwhile, expression of CD206 (E1, E2, E3) and IL10 (F1, F2), as well as arginase activity (G), was hardly response to IL-4 induction. E3: Representative products of western blot analysis for CD206. All bar graphs reported mean \pm SD for six experiments performed in triplicate except E1 \& E3 (three experiments). ${ }^{*} p<0.05$ vs control under basal condition, ${ }^{*} p<0.01$ vs control under LPS or IL-4 stimulation.

after stimulation with LPS and IL-4 for 24 hours, respectively. As shown in Figure 2 A, B, C, D, expression of TNF $\alpha$, IL-6, and IL-12p40 at mRNA (Fig. 2 A1, B1, C1) and protein (Fig. 2 A2, B2, C2) levels, as well as the expression of iNOS (Fig. 2D1) and production of NO (Fig. 2D2), was significantly increased in response to LPS stimulation in macrophages treated with RRM rats sera compared with sham rats sera. In contrast, expression of CD206 (Fig. 2 E1, E2, and 


\section{Cellular Physiology Cell Physiol Biochem 2015;36:358-372 \begin{tabular}{l|l|l|}
\cline { 2 - 3 } DOI: 10.1159/000430106 & O 2015 S. Karger AG, Bas
\end{tabular} \\ Li et al.: Macrophage Polarization in CKD}

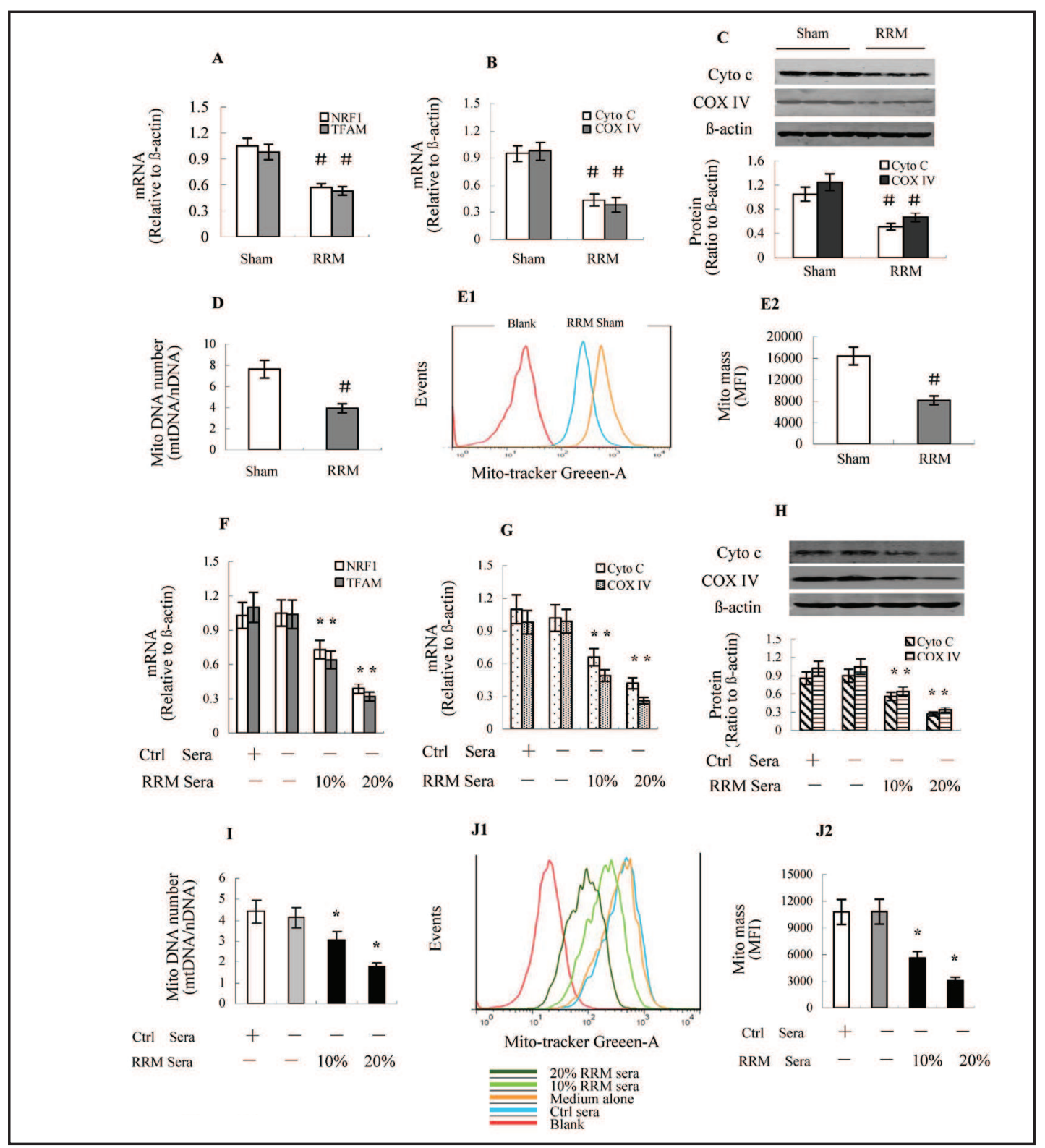

Fig. 3. Mitochondrial biogenesis in peritoneal macrophages under the condition of CKD. Mitochondrial biogenesis in peritoneal macrophages of RRM rats (A-E) and normal rats treated with RRM rats' sera (F-J) was shown. Peritoneal macrophages were isolated from RRM rats and subjected to analysis for the markers of mitochondrial biogenesis. Expression of NRF1 and TFAM (A), and cytochrome $\mathrm{C}$ and cytochrome $\mathrm{C}$ oxidase IV (B, C), and mitochondrial DNA number (D), as well as mitochondrial mass (E1, E2) were significantly reduced in peritoneal macrophages of RRM rats compared with sham rats. In another experiments, peritoneal macrophages were isolated from normal rats and treated with RRM rats-derived sera for 24 hours (F-J). Then mitochondrial biogenesis was determined. Treatment with RRM rat's sera reduced expression of NRF1 and TFAM (F), cytochrome $C$ and cytochrome $C$ oxidase IV (G, H), and mitochondrial DNA number (I), as well as mitochondrial mass (J1, J2), in a concentration-dependent way. E1, J1: Representative analysis of flow cytometer for mitochondrial mass with Mito-tracker. All bar graphs reported mean \pm SD for six experiments performed in triplicate except $\mathrm{C}$ and $\mathrm{H}$ ( three experiments). ${ }^{*} p<0.01$ vs control, ${ }^{*} p<0.01$ vs sham. ANOVA, $p<0.05$ (F-J2).

E3) and IL-10 (Fig. 2 F1 and F2), as well as arginase activity (Fig. 2G), was hardly response to IL-4 induction in macrophages treated with RRM rats sera. 


\section{Cellular Physiology Cell Physiol Biochem 2015;36:358-372 \begin{tabular}{l|l|l}
\hline DOI: 10.1159/000430106 & (c)15 S. Karger AG, Basel
\end{tabular} \begin{tabular}{l|l} 
and Biochemistry Published online: May 06, 2015 & www.karger.com/cpb \\
\hline
\end{tabular} \\ Li et al.: Macrophage Polarization in CKD}

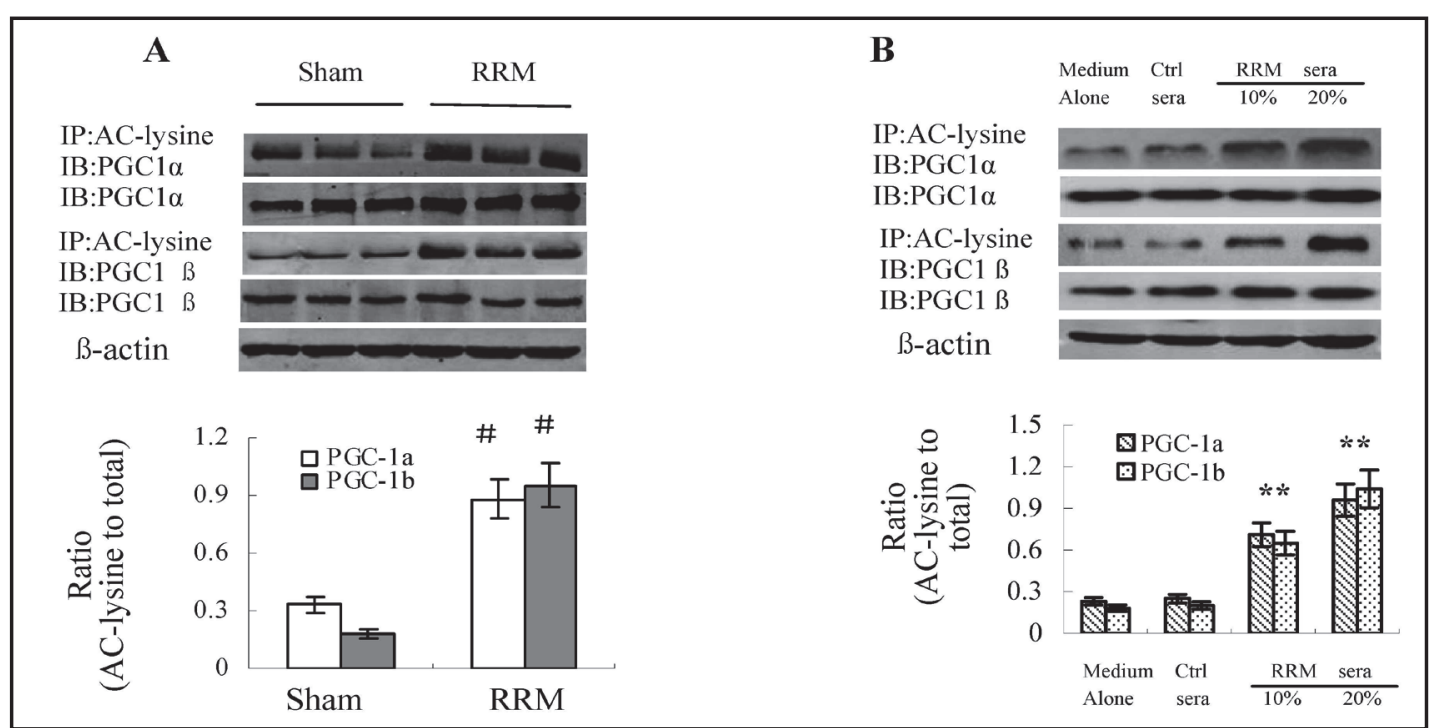

Fig. 4. Acetylation of PGC- $1 \alpha$ and PGC-1ß in peritoneal macrophages under the condition of CKD. Peritoneal macrophages were isolated from RRM rats and then expression and acetylation of PGC- $1 \alpha$ and PGC-1ß were determined. Acetylation of PGC- $1 \alpha$ and PGC- $1 ß$ was significantly increased in the cells from RRM rats compared with sham rats (A). In another experiments, expression and acetylation of PGC- $1 \alpha$ and PGC-1ß were determined in peritoneal macrophages from normal rats followed by treatment with different concentration of RRM rats' sera for 24 hours (B). Treatment with RRM rats' sera led to increased acetylation in a concentration-dependent way. All bar graphs reported mean \pm SD for three experiments performed in triplicate. ${ }^{*} p<0.01$ vs control, ${ }^{*} p<0.01$ vs sham. ANOVA, $p<0.05$ (B).

Taken together, CKD enhanced macrophage M1 and impaired M2 polarization.

\section{CKD inhibited macrophage mitochondrial biogenesis}

It has been demonstrated that mitochondrial biogenesis is closely related with macrophage polarization $[15,26]$. To validate these findings in our model system, we evaluated mitochondrial biogenesis in macrophages derived from RRM rats. As shown in Figure $3 \mathrm{~A}-\mathrm{E}$, expression of NRF1 and TFAM (the essential transcriptional factors for mitochondrial biogenesis) (Fig. 3A), cytochrome C and cytochrome C oxidase IV (COX IV) (the specific mitochondrial proteins) (Fig. $3 \mathrm{~B}$ and C) was significantly reduced. In agreement with these results, impaired mitochondrial biogenesis was further confirmed by reduced mitochondrial DNA number (Fig. 3D) and mitochondrial mass measured by flow cytometer with Mitochondrial probe (Fig. 3 E1 and E2).

We also evaluated the effect of RRM rats' sera on mitochondrial biogenesis in normal macrophages. Peritoneal macrophages were isolated from normal rats and treated with different concentration of RRM rats-derived sera (10\%, and 20\%) for 24 hours. Sera derived from sham rats were used as the control. As shown in Figure 3F-J, treatment with uremic sera led to significant reduction in mitochondrial biogenesis as evidenced by reduced expression of NRF1 and TFAM (Fig. 3F), cytochrosome C and cytochrome C oxidase IV (Fig. 3G and H), and reduced mitochondrial DNA (Fig. 3I) and mass (Fig. 3 J1 and J2).

To further confirm the reduced mitochondrial biogenesis in macrophages, we analyzed expression and acetylation of peroxisome proliferator activated receptor gamma coactivator1(PGC-1), a master regulator of mitochondrial biogenesis [27]. In consistent with early reports $[15,28]$, both PGC- $1 \alpha$ and PGC-1ß were identified in macrophages (Fig. 4). As shown in Figure 4 A, expression of PGC- $1 \alpha$ and PGC- $1 ß$ was comparable in macrophages between RRM rats and sham rats. However, acetylation of PGC- $1 \alpha$ and PGC-1ß, a key way to inhibit PGC-1 activity [29, 30], was significantly increased in RRM rats (Fig. 4A). Similar results were observed in normal peritoneal macrophages treated with RRM rats' sera (Fig. 4B). 


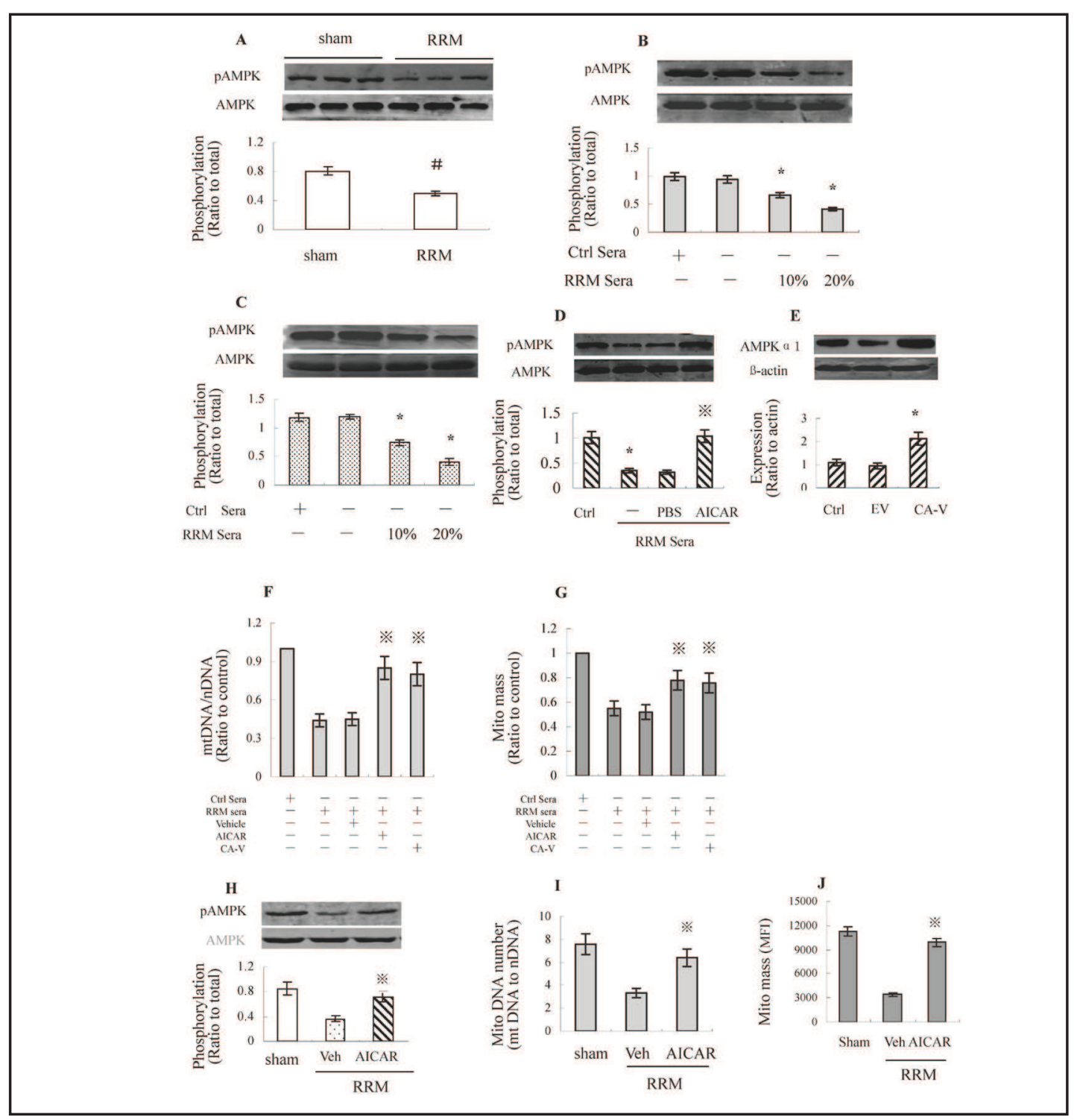

Fig. 5. Involvement of AMPK in reduced mitochondrial biogenesis in macrophages under the condition of CKD. Phosphorylation of AMPK was determined in peritoneal macrophages from RRM rats (A). Phosphorylation of AMPK was also evaluated in peritoneal (B) and bone marrow-derived (C) macrophages from normal rats followed by treatment with different concentration of RRM rats' sera for 24 hours. To evaluate the involvement of AMPK in mitochondrial biogenesis, peritoneal macrophages from normal rats were stimulated with AICAR (1mM) for 2 hours and then incubated with RRM rats' sera (20\%) for additional 24 hours. Treatment with AICAR increased phosphorylation of AMPK (D). The activation of AMPK was also enhanced by over-expression of constitutively active AMPK $\alpha 1$ which was confirmed by western blot (E). Peritoneal macrophages transfected with empty vector (EV) or constitutively active (CA) $\alpha 1$ AMPK adenovirus were incubated with RRM rats' sera (20\%) for 24 hours. Treatment with AICAR and over-expression of constitutively active (CA) $\alpha 1$ AMPK could block the inhibitory effect of RRM rats' sera on mitochondrial biogenesis as revealed by increased mitochondrial DNA copy (F) and mass (G), respectively. In another experiment, RRM rats were treated with intraperitoneal injection of AICAR or vehicle for one week and then peritoneal macrophages were isolated for the analysis. Treatment with AICAR enhanced activation of AMPK $(\mathrm{H})$, which was associated with increased mitochondrial DNA number (I) and mitochondrial mass (J) in ex vivo macrophages from RRM rats. All bar graphs reported mean \pm SD for three experiments performed in triplicate except F, G, I, and J (six experiments). ${ }^{*} p<0.01$ vs control, ${ }^{\circledR} p<0.01$ vs sham, ${ }^{*} p<0.01$ vs vehicle. ANOVA, $p<0.05(\mathrm{~B}, \mathrm{C})$. 


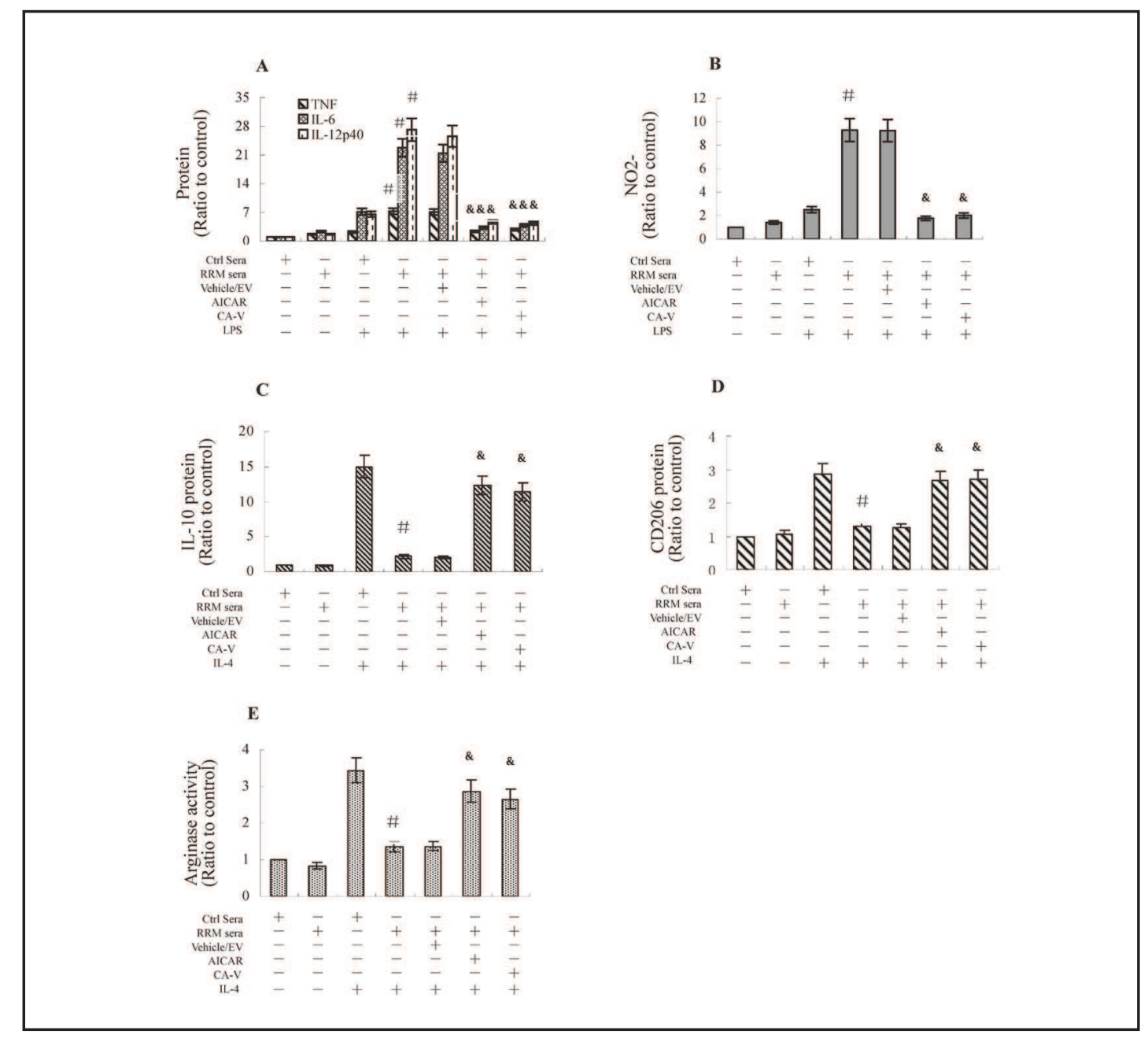

Fig. 6. Enhancing AMPK activation restored M2 polarization. To enhance the activation of AMPK, peritoneal macrophages from normal rats were stimulated with AICAR $(1 \mathrm{mM})$ and transfected with constitutively active (CA) $\alpha 1$ AMPK adenovirus, respectively. The cells were then incubated with RRM rats' sera (20\%) for additional 24 hours. After removal of sera, the cells were subjected to analysis of M1 and M2 markers followed by LPS and IL-4 stimulation for 24 hours, respectively. Treatment with both AICAR and over-expression of constitutively active (CA) $\alpha 1$ AMPK could inhibit LPS-induced M1 polarization revealed by inhibited expression of TNF $\alpha$, IL-6, and IL-12p40 (A), and production of NO (B). Treatment with both AICAR and over-expression of constitutively active (CA) $\alpha 1$ AMPK restored IL-4-induced M2 polarization detected by increased expression of IL-10 (C), CD206 (D), and arginase activity (E). All bar graphs reported mean \pm SD for six experiments except D (three experiments) performed in triplicate. ${ }^{\circledR} p<0.01$ vs control under LPS or IL-4 stimulation, ${ }^{\circledR} p<0.01$ vs vehicle. (EV, empty vector).

These data suggested that mitochondrial biogenesis was inhibited under the condition of CKD.

CKD disturbed macrophage polarization and mitochondrial biogenesis via inhibition of $A M P K$

AMPK has been demonstrated to play a key role in cellular energy homeostasis and influence macrophage phenotypic transition $[17,19,31]$. To exam the involvement of AMPK in macrophages dysfunctions observed under the condition of CKD, we measured phosphorylation form of AMPK. As shown in Figure 5 A, phosphorylation of AMPK was significantly reduced in peritoneal macrophages from RRM rats compared with sham rats. Moreover, treatment with RRM rats' sera resulted in significant reduction in phosphorylation 


\section{Cellular Physiology Cell Physiol Biochem 2015;36:358-372 \\ \begin{tabular}{ll|l} 
and Biochemistry $10.1159 / 000430106$ & $\begin{array}{l}\text { Dublished online: May 06, } 2015 \\
\text { www.karger.com/cpb }\end{array}$ \\
\cline { 2 - 3 }
\end{tabular}}

of AMPK in both peritoneal macrophages (Fig. 5B) and BMDMs (Fig. 5C) from normal rats. These data suggested that the activity of AMPK was inhibited in macrophages under the condition of CKD. To further confirm the role of AMPK in disorder of macrophage polarization, we used 5-aminoimidazole-4-carbox-amide-1- $\beta$-d-ribofuranoside (AICAR, $1 \mathrm{mM}$ ), a small molecular kinase activator [32], (Fig. 5D), or genetic over-expression of constitutively active AMPK $\alpha 1$ (Fig. 5E) to enhance activation of AMPK in normal macrophages before RRM rats' sera treatment. As presented in Figure 5 F-G, both AICAR and over-expression of constitutively active AMPK 1 blocked the inhibitory effect of RRM rats' sera on mitochondrial biogenesis as evidenced by restored mitochondrial DNA number (Fig. 5F) and mass (Fig. 5G). To test the effect of activation of AMPK on mitochondrial biogenesis in ex vivo macrophages, we treated RRM rats with intraperitoneal injection of AICAR or vehicle for one week and then isolated peritoneal macrophages for the analysis. As shown in Figure $5 \mathrm{H}-\mathrm{J}$, treatment with AICAR increased activation of AMPK (H), which was associated with increased mitochondrial DNA number (I), and mitochondrial mass (J). Moreover, treatment with AICAR or overexpression of constitutively active AMPK $\alpha 1$ inhibited M1 polarized activation in response to LPS stimulation (Fig. 6A and B), while restored M2 polarized activation in response to IL-4 induction (Fig. 6 C, D and E).

These data suggested disturbed macrophage polarization and impaired mitochondrial biogenesis were linked with inhibition of AMPK under the condition of CKD.

\section{Discussion}

Chronic systemic inflammation is characteristic feature of CKD [33]. Macrophages, the central mediators of inflammatory responses, contribute both to the initiation and the resolution of inflammation. Given the potential roles of macrophage polarization in a broad spectrum of biological processes in health and disease, it is essential to understand how macrophage polarization could be modulated under a different pathology condition. Data presented in this report showed that macrophages from rats with CKD or cultured under the condition of uremia had enhanced M1 polarization and impaired M2 polarization. Furthermore, the disturbed macrophage polarization was associated with reduced mitochondrial biogenesis and AMPKactivity. Enhancing activation of AMPK could significantly restore mitochondrial biogenesis and M2 macrophage polarization. Our data provided the first evidence showing chronic renal failure could affect macrophage polarization which was linked to reduced activation of AMPK and mitochondrial biogenesis. In a mouse model of unilateral ureteral obstruction, renal macrophage polarization is demonstrated to be modulated by a renal resident cell [34]. These data might provide a novel therapeutic strategy for the prevention and treatment of chronic systemic inflammation and renal inflammation in patients with CKD.

Diversity and plasticity are two hallmarks of macrophages. Several studies have shown uncontrolled macrophage polarization is associated with pathological conditions $[5,7,8]$. In this study, we presented several lines of evidence showing that macrophage polarization was disturbed under the condition of CKD. M1 and M2 polarization was induced in peritoneal and bone marrow derived macrophages from CKD rats and normal rats. The markers of M1 polarization were significantly increased in response to LPS stimulation in all macrophages either from CKD rats or from normal rats cultured under the condition of uremia. Meanwhile, the markers of M2 polarization were hardly response to IL-4 induction. These data suggested that CKD promoted macrophage M1 polarization and impaired M2 polarization. Since M1 polarized macrophages produce pro-inflammatory cytokines and are potentiated to proinflammatory stimuli $[15,35]$, it is reasonable to propose that uncontrolled macrophage polarization might be an important mechanism for chronic inflammation observed in CKD. In accordance with this information, the main causes of death in patients with CKD are related to infectious and cardiovascular diseases which are closely linked to macrophage perturbation [36]. 


\section{Cellular Physiology Cell Physiol Biochem 2015;36:358-372 \\ \begin{tabular}{ll|l} 
and Biochemistry $10.1159 / 000430106$ & $\begin{array}{l}\text { Dublished online: May 06, } 2015 \\
\text { Po } 2015 \text { S. Karger AG, Basel } \\
\text { www.karger.com/cpb }\end{array}$ \\
\hline
\end{tabular}}

Mitochondrial biogenesis has been shown to play an important role in macrophage polarization $[15,26]$. Inhibition of mitochondrial biogenesis is associated with abnormal macrophage polarization. Our data showed that macrophages from either RRM rats or cultured under the condition of uremia had reduced mitochondrial biogenesis as revealed by reduced expression of NRF1 and TAMF, expression of mitochondrial specific proteins, and mitochondrial DNA and mass. Moreover, the master regulator of mitochondrial biogenesis, PGC-1, was inhibited as detected by increased acetylation which is a key way to inhibit its activity. In consistent with these observations, expression of PGC- 1 and NRF-1 are down regulated in blood monocytes in CKD patients [37], suggesting that mitochondrial biogenesis is inhibited. Macrophages with reduced mitochondrial content are more likely to be M1 polarization and display increased sensitivity to inflammatory stimuli. Indeed, macrophages cultured under the condition of uremic toxin produce higher level of pro-inflammatory cytokines in response to LPS [38].

The mechanisms for disturbed macrophage polarization and inhibited mitochondrial biogenesis under the condition of CKD remain unclear. Several evidence in this study showed that inhibition of AMPK might play a key role in this process. Firstly, we detected lower levels of AMPK activity in macrophages from CKD rats and cultured under the microenvironments of CKD. Secondly, enhancing AMPK activation resulted in restore of mitochondrial biogenesis and M2 polarization, while inhibition of M1 polarization. In accordance with these observations, deletion of AMPK led to decreased mitochondrial content and M2 polarization, while increased M1 polarization [39]. Conversely, activation of AMPK was reported to have anti-inflammatory properties both in vitro and in vivo $[19,32]$. Our results were also consistent with early report that AMPK can affect PGC-1 activity [17, 40]. The molecular mechanisms for inhibition of AMPK and AMPK-mediated macrophage perturbation under the condition of CKD warrant further investigation.

Activation and polarization of macrophages can be induced by diverse mediators [41]. In the present study, LPS was used for the generation of polarized macrophage populations. Moreover, we used ex vivo macrophages derived from RRM rats or macrophages cultured with CKD sera. CKD can lead to the retention of a myriad of compounds (also called uremic toxins) that under normal condition are excreted by the healthy kidneys. Even though major advance has been made in identification and characterization of uremic toxins and the knowledge of their pathophysiologic importance [42], the compounds were responsible for macrophages perturbation observed in this study remained to be further studied. Both macrophage and dendritic cells belong to the mononuclear phagocyte system which is a family cells derived from a committed progenitor in the bone marrow and play an essential role in the regulation of immune integrity. Considering metabolic reprogramming occurs during activation of dendritic cells [43], whether chronic kidney disease affects their mitochondrial biogenesis and activation needs further investigation.

In summary, macrophages from CKD rats and cultured under the condition of CKD had enhanced M1 and impaired M2 polarization and reduced mitochondrial biogenesis. Enhancing AMPK activation restored mitochondrial biogenesis and M2 polarization. This provides a potential therapeutic strategy for the intervention of chronic inflammation in patients with CKD.

\section{Acknowledgements}

This work was supported National Nature and Science Grant (No.81270827) to Dr. Q. G. Zhou.

\section{Disclosure Statement}

No. 


\section{Cellular Physiology Cell Physiol Biochem 2015;36:358-372 \begin{tabular}{c|l}
\hline DOI: 10.1159/000430106 & (c) 2015 S. Karger AG, Basel
\end{tabular} \begin{tabular}{l|l} 
and Biochemistry Published online: May 06, 2015 & www.karger.com/cpb \\
\hline
\end{tabular}}

Li et al.: Macrophage Polarization in CKD

\section{Reference}

1 Recio-Mayoral A, Banerjee D, Streather C, Kaski JC: Endothelial dysfunction, inflammation and atherosclerosis in chronic kidney disease--a cross-sectional study of predialysis, dialysis and kidneytransplantation patients. Atherosclerosis 2011;216:446-451.

2 Shlipak MG, Fried LF, Cushman M, Manolio TA, Peterson D, Stehman-Breen C, Bleyer A, Newman A, Siscovick D, Psaty B: Cardiovascular mortality risk in chronic kidney disease: comparison of traditional and novel risk factors. JAMA 2005;293:1737-1745.

3 Lawrence T, Natoli G: Transcriptional regulation of macrophage polarization: enabling diversity with identity. Nat Rev Immunol 2011;11:750-761.

4 Sica A, Mantovani A: Macrophage plasticity and polarization: in vivo veritas. J Clin Invest 2012;122:787795.

5 Mosser DM, Edwards JP: Exploring the full spectrum of macrophage activation. Nat Rev Immunol 2008;8:958-969.

6 Gordon S, Martinez FO: Alternative activation of macrophages: mechanism and functions. Immunity 2010;32:593-604.

7 Lumeng CN, Bodzin JL, Saltiel AR: Obesity induces a phenotypic switch in adipose tissue macrophage polarization. J Clin Invest 2007;117:175-184.

8 Stoger JL, Gijbels MJ, van der Velden S, Manca M, van der Loos CM, Biessen EA, Daemen MJ, Lutgens E, de Winther MP: Distribution of macrophage polarization markers in human atherosclerosis. Atherosclerosis 2012;225:461-468.

9 Lech M, Grobmayr R, Ryu M, Lorenz G, Hartter I, Mulay SR, Susanti HE, Kobayashi KS, Flavell RA, Anders HJ: Macrophage phenotype controls long-term AKI outcomes--kidney regeneration versus atrophy. J Am Soc Nephrol 2014;25:292-304.

10 Anders HJ, Ryu M: Renal microenvironments and macrophage phenotypes determine progression or resolution of renal inflammation and fibrosis: Kidney Int 2011;80:915-925.

11 Rohas LM, St-Pierre J, Uldry M, Jager S, Handschin C, Spiegelman BM: A fundamental system of cellular energy homeostasis regulated by PGC-1alpha. Proc Natl Acad Sci U S A 2007;104:7933-7938.

12 Michel S, Wanet A, De Pauw A, Rommelaere G, Arnould T, Renard P: Crosstalk between mitochondrial (dys) function and mitochondrial abundance. J Cell Physiol 2012;227:2297-2310.

13 Rodriguez-Prados JC, Traves PG, Cuenca J, Rico D, Aragones J, Martin-Sanz P, Cascante M, Bosca L: Substrate fate in activated macrophages: a comparison between innate, classic, and alternative activation. J Immunol 2010;185:605-614.

14 Pearce EL, Pearce EJ: Metabolic pathways in immune cell activation and quiescence. Immunity 2013;38:633-643.

15 Vats D, Mukundan L, Odegaard JI, Zhang L, Smith KL, Morel CR, Wagner RA, Greaves DR, Murray PJ, Chawla A: Oxidative metabolism and PGC-1beta attenuate macrophage-mediated inflammation. Cell Metab 2006;4:13-24.

16 Date D, Das R, Narla G, Simon DI, Jain MK, Mahabeleshwar GH: Kruppel-like transcription factor 6 regulates inflammatory macrophage polarization. J Biol Chem 2014;289:10318-10329.

17 Canto C, Gerhart-Hines Z, Feige JN, Lagouge M, Noriega L, Milne JC, Elliott PJ, Puigserver P, Auwerx J: AMPK regulates energy expenditure by modulating NAD+ metabolism and SIRT1 activity. Nature 2009;458:10561060.

18 Canto C, Jiang LQ, Deshmukh AS, Mataki C, Coste A, Lagouge M, Zierath JR, Auwerx J: Interdependence of AMPK and SIRT1 for metabolic adaptation to fasting and exercise in skeletal muscle. Cell Metab 2010;11:213-219.

19 Sag D, Carling D, Stout RD, Suttles J: Adenosine 5'-monophosphate-activated protein kinase promotes macrophage polarization to an anti-inflammatory functional phenotype. J Immunol 2008;181:8633-8641.

20 Giulivi C, Ross-Inta C, Omanska-Klusek A, Napoli E, Sakaguchi D, Barrientos G, Allen PD, Pessah IN: Basal bioenergetic abnormalities in skeletal muscle from ryanodine receptor malignant hyperthermiasusceptible R163C knock-in mice. J Biol Chem 2011;286:99-113.

21 Arranz A, Doxaki C, Vergadi E, Martinez de la Torre Y, Vaporidi K, Lagoudaki ED, Ieronymaki E, Androulidaki A, Venihaki M, Margioris AN, Stathopoulos EN, Tsichlis PN, Tsatsanis C: Akt1 and Akt2 protein kinases differentially contribute to macrophage polarization. Proc Natl Acad Sci U S A 2012;109:9517-9522.

22 Zhou QG, Fu XJ, Xu GY, Cao W, Liu HF, Nie J, Liang M, Hou FF: Vascular insulin resistance related to endoplasmic reticulum stress in aortas from a rat model of chronic kidney disease. Am J Physiol Heart Circ Physiol 2012;303:H1154-1165.

23 Woldt E, Sebti Y, Solt LA, Duhem C, Lancel S, Eeckhoute J, Hesselink MK, Paquet C, Delhaye S, Shin Y, Kamenecka TM, Schaart G, Lefebvre P, Neviere R, Burris TP, Schrauwen P, Staels B, Duez H: Rev-erb-alpha 


\section{Cellular Physiology Cell Physiol Biochem 2015;36:358-372 \begin{tabular}{l|l} 
DOI: 10.1159/000430106 & $\begin{array}{l}\text { C 2015 S. Karger AG, Basel } \\
\text { www.karger.com/cpb }\end{array}$ \\
\hline
\end{tabular} \\ Li et al.: Macrophage Polarization in CKD}

modulates skeletal muscle oxidative capacity by regulating mitochondrial biogenesis and autophagy. Nat Med 2013;19:1039-1046.

24 Wen H, Gris D, Lei Y, Jha S, Zhang L, Huang MT, Brickey WJ, Ting JP: Fatty acid-induced NLRP3-ASC inflammasome activation interferes with insulin signaling. Nat Immunol 2011;12:408-415.

25 Mantovani A, Sica A, Sozzani S, Allavena P, Vecchi A, Locati M: The chemokine system in diverse forms of macrophage activation and polarization. Trends Immunol 2004;25:677-686.

26 MacGarvey NC, Suliman HB, Bartz RR, Fu P, Withers CM, Welty-Wolf KE, Piantadosi CA: Activation of mitochondrial biogenesis by heme oxygenase-1-mediated NF-E2-related factor-2 induction rescues mice from lethal Staphylococcus aureus sepsis. Am J Respir Crit Care Med 2012;185:851-861.

27 Lin J, Handschin C, Spiegelman BM: Metabolic control through the PGC-1 family of transcription coactivators. Cell Metab 2005;1:361-370.

28 McCarthy C, Lieggi NT, Barry D, Mooney D, de Gaetano M, James WG, McClelland S, Barry MC, EscoubetLozach L, Li AC, Glass CK, Fitzgerald DJ, Belton O: Macrophage PPAR gamma Co-activator-1 alpha participates in repressing foam cell formation and atherosclerosis in response to conjugated linoleic acid. EMBO Mol Med 2013;5:1443-1457.

29 Kelly TJ, Lerin C, Haas W, Gygi SP, Puigserver P: GCN5-mediated transcriptional control of the metabolic coactivator PGC-1beta through lysine acetylation. J Biol Chem 2009;284:19945-19952.

30 Sakai M, Matsumoto M, Tujimura T, Yongheng C, Noguchi T, Inagaki K, Inoue H, Hosooka T, Takazawa K, Kido Y, Yasuda K, Hiramatsu R, Matsuki Y, Kasuga M: CITED2 links hormonal signaling to PGC-1alpha acetylation in the regulation of gluconeogenesis. Nat Med 2012;18:612-617.

31 Mounier R, Theret M, Arnold L, Cuvellier S, Bultot L, Goransson O, Sanz N, Ferry A, Sakamoto K, Foretz M, Viollet B, Chazaud B: AMPKalpha1 regulates macrophage skewing at the time of resolution of inflammation during skeletal muscle regeneration. Cell Metab 2013;18:251-264.

32 Hoogendijk AJ, Pinhancos SS, van der Poll T, Wieland CW: AMP-activated protein kinase activation by 5-aminoimidazole-4-carbox-amide-1-beta-D-ribofuranoside (AICAR) reduces lipoteichoic acid-induced lung inflammation. J Biol Chem 2013;288:7047-7052.

33 Heine GH, Ortiz A, Massy ZA, Lindholm B, Wiecek A, Martinez-Castelao A, Covic A, Goldsmith D, Suleymanlar G, London GM, Parati G, Sicari R, Zoccali C, Fliser D: Monocyte subpopulations and cardiovascular risk in chronic kidney disease. Nat Rev Nephrol 2012;8:362-369.

34 Fujiu K, Manabe I, Nagai R: Renal collecting duct epithelial cells regulate inflammation in tubulointerstitial damage in mice. J Clin Invest 2011;121:3425-3441.

35 Festuccia WT, Pouliot P, Bakan I, Sabatini DM, Laplante M: Myeloid-specific Rictor deletion induces M1 macrophage polarization and potentiates in vivo pro-inflammatory response to lipopolysaccharide. PloS one 2014;9:e95432.

36 Wen CP, Cheng TY, Tsai MK, Chang YC, Chan HT, Tsai SP, Chiang PH, Hsu CC, Sung PK, Hsu YH, Wen SF: Allcause mortality attributable to chronic kidney disease: a prospective cohort study based on 462293 adults in Taiwan. Lancet 2008;371:2173-2182.

37 Zaza G, Granata S, Masola V, Rugiu C, Fantin F, Gesualdo L, Schena FP, Lupo A: Downregulation of nuclear-encoded genes of oxidative metabolism in dialyzed chronic kidney disease patients. PloS one 2013;8:e77847.

38 Adesso S, Popolo A, Bianco G, Sorrentino R, Pinto A, Autore G, Marzocco S: The uremic toxin indoxyl sulphate enhances macrophage response to LPS. PloS one 2013;8:e76778.

39 Galic S, Fullerton MD, Schertzer JD, Sikkema S, Marcinko K, Walkley CR, Izon D, Honeyman J, Chen ZP, van Denderen BJ, Kemp BE, Steinberg GR: Hematopoietic AMPK beta1 reduces mouse adipose tissue macrophage inflammation and insulin resistance in obesity. J Clin Invest 2011;121:4903-4915.

40 Li L, Pan R, Li R, Niemann B, Aurich AC, Chen Y, Rohrbach S: Mitochondrial biogenesis and peroxisome proliferator-activated receptor-gamma coactivator-1alpha (PGC-1alpha) deacetylation by physical activity: intact adipocytokine signaling is required. Diabetes 2011;60:157-167.

41 Murray PJ, Allen JE, Biswas SK, Fisher EA, Gilroy DW, Goerdt S, Gordon S, Hamilton JA, Ivashkiv LB, Lawrence T, Locati M, Mantovani A, Martinez FO, Mege JL, Mosser DM, Natoli G, Saeij JP, Schultze JL, Shirey KA, Sica A, Suttles J, Udalova I, van Ginderachter JA, Vogel SN, Wynn TA: Macrophage activation and polarization: nomenclature and experimental guidelines. Immunity 2014;41:14-20.

42 Vanholder R, Van Laecke S, Glorieux G: What is new in uremic toxicity? Pediatr Nephrol 2008;23:12111221.

43 Everts B, Amiel E, Huang SC, Smith AM, Chang CH, Lam WY, Redmann V, Freitas TC, Blagih J, van der Windt GJ, Artyomov MN, Jones RG, Pearce EL, Pearce EJ: TLR-driven early glycolytic reprogramming via the kinases TBK1-IKKvarepsilon supports the anabolic demands of dendritic cell activation. Nat Immunol 2014;15:323-332. 